\title{
Versuche zur Brutaufzucht der Seezunge Solea solea in kleinen Aquarien
}

\author{
JÜRGEN FLÜCHTER \\ Biologische Anstalt Helgoland, Meeresstation, Helgoland
}

\begin{abstract}
Experiments concerning the offspring raising of the sole Solea solea (L.) in small aquaria. Soles, both freshly caught in the North Sea in May 1965 and living in the laboratory for several months, spawned successfully in large laboratory concrete tanks. Mortality of offspring was about $20 \%$ and reached its maximum during the 11 th to 13 th day after hatching. The larvae approach the bottom when their yolk sac is absorbed. Even when they are still quite symmetrical, they begin to lie down on their sides for various lengths of time. During this intermediate phase they straighten up and swim with their backs up in the normal upright position each time they are disturbed or about to catch food. Permanent side lying and swimming begins only 7 days later. During growth the larvae change their color several times. This color change may prove a useful tool for determining developmental stages and age groups of soles in the open sea. With the occurrence of light pigments at an age of 4 to 5 weeks, the young soles respond to artificially created water currents by clinging to the substrate. Growth rates of soles kept in small containers up to an age of 6 months compared well with those of individuals caught in the open sea. A method for mass-culture of the brine shrimp Artemia salina is described.
\end{abstract}

\section{EINLEITUNG}

Die Aufzucht der Larven von Meeresfischen unter Laboratoriumsbedingungen bereitet beträchtliche Schwierigkeiten. Von einer Zucht über mehrere Generationen gar nicht zu reden, belastet bereits die sehr hohe Larvensterblichkeit aller bislang untersuchten Arten, insbesondere am Ende der Dottersackphase, die Ergebnisse der Laboruntersuchungen mit einem bedeutenden Unsicherheitsfaktor. In der Meeresstation der Biologischen Anstalt Helgoland gelang es nunmehr erstmals, ein relativ befriedigendes Aufzuchtverfahren für Seezungen [Solea solea (L); sgn. S. vulgaris Quensel] zu entwickeln. Leider ist das gegenwärtig zur Verfügung stehende Zahlenmaterial noch gering. Die erzielten Versuchsergebnisse rechtfertigen jedoch - insbesondere im Hinblick auf unsere noch recht mangelhaften einschlägigen Kenntnisse - eine kurze Darstellung der bisher gewonnenen Erkenntnisse. Insbesondere werden einige Beobachtungen über die bisher wenig bekannte Lebensgeschichte der jüngsten Stadien mitgeteilt. Zweifellos dürfte die Seezunge für die Bearbeitung experimentell-ökologischer Fragestellungen im Labor besonders geeignet sein. 


\section{GEWINNUNG DES EIMATERIALS}

Am 14. Mai 1965 wurde bei Helgoland (Position $54^{\circ} 15^{\prime} \mathrm{N}, 7^{\circ} 46^{\prime} \mathrm{O}$ ) mit dem Kuttertrawl eine Anzahl von Seezungen Solea solea (L.) zwischen 30 und $37 \mathrm{~cm}$ Totallänge gefangen. Die Wassertemperatur am Boden betrug $8^{\circ} \mathrm{C}$. Eine Präparation von drei 우 zeigte durchweg das Bild des Reifestadiums V (Ovar hellorange, nur einzelne klare Eier). Die Tiere wurden in ein 1 Meter tiefes, $4 \mathrm{~m}^{3}$ Wasser fassendes Bassin gebracht, wo sie in der Nacht vom 18. zum 19. Mai bei $12^{\circ} \mathrm{C}$ erstmals ablaichten. Das einzelne $q$ gab seinen Eivorrat nicht auf einmal, sondern wie an dem sukzessive schwindenden Laichansatz ersichtlich war, in mindestens drei bis vier Schüben mit jeweils etwa 3 Tagen Abstand ab. Das Laichen fand stets während der Nachtstunden statt. Das sukzessive Reifen der Eier sowie die Kleinheit der männlichen Goaaden lassen ein Abstreifen der Laichprodukte und eine künstliche Besamung, wie sie

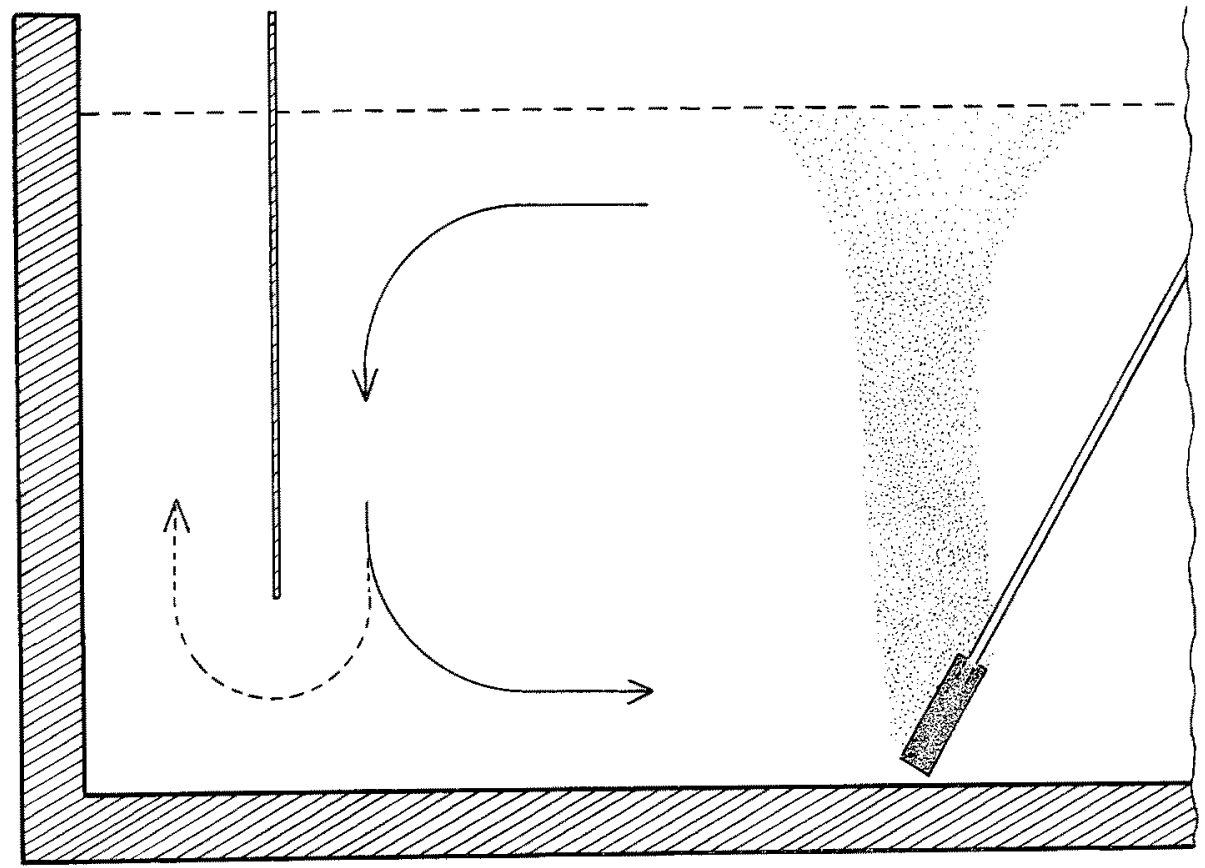

Abb. 1: Schematische Darstellung der Methode des Abfangens der Seezungeneier aus dem Laichbedken

vielfach in der Fischzucht vorgenommen wird, nicht zu. Auch in der offenen See scheint das Reifen der Eier schubweise vor sich zu gehen. Fließend reife $q$ (Stadium VI) werden auffällig selten gefangen (BüCKMANN 1934).

Einige seit September des Vorjahres zu Markierungs- und Wachstumsuntersuchungen in einem etwa gleichgroßen Becken gehälterte und mit gekochten Miesmuscheln gefütterte Seezungen von 27 bis $35 \mathrm{~cm}$ Länge wurden ebenfalls reif und laichten, aller- 
dings erst in den ersten Junitagen bei einer Wassertemperatur von $15^{\circ} \mathrm{C}$, erfolgreich ab. Dieses Laichreifwerden in der Gefangenschaft läßt eine Zucht der Seezunge über mehrere Generationen möglich erscheinen. Bislang war man bei Aufzuchtversuchen mit Seezungenbrut darauf angewiesen, einzelne Eier aus Planktonfängen zu isolieren (Fabre-Domergue \& Biétrix 1905).

Die im Wasser frei schwebenden Eier konnten in folgender Weise in den Ecken des Beckens angereichert werden: jede Ecke wurde durch eine Scheidewand vom übrigen Wasserkörper abgetrennt, jedoch in Bodennähe ein etwa $30 \mathrm{~cm}$ breiter Raum freigelassen. Bei in der Mitte des Hauptbeckens angebrachter starker Durchlüftung gerieten die Eier nach und nach in den strömungstoten Raum unterhalb der Scheidewand und stiegen in den "Ecken" auf, wo sie oben abgeschöpft werden konnten (Abb. 1). Bei einem Salzgehalt von $35 \%$ hatten die Eier einen Durchmesser von 1,12 bis $1,27 \mathrm{~mm}$.

\section{ERBRUTUNG UND AUFZUCHT}

Da es über die Aufzucht von Seezungenlarven nur wenige Anhaltspunkte gibt (FAbre-Domergue \& Biétrix 1905), wurden die Eier in kleinen Portionen einer Anzahl verschiedener Bedingungskombinationen unterworfen. Lediglich folgendes Verfahren führte zu einem zufriedenstellenden Erfolg: ein Vollglasbecken, $40 \mathrm{~cm}$ lang, $25 \mathrm{~cm}$ breit und $30 \mathrm{~cm}$ tief wurde als Innenfilter eingerichtet (FLÜCHTER 1964) und in ein größeres, dunkelwandiges Gefäß mit durchfließendem Wasser bestimmter Temperatur eingesetzt. Als Lichtquelle war eine Leuchtstoffröhre in $30 \mathrm{~cm}$ Abstand über der Wasseroberfläche des Beckens angebracht. An der Wasseroberfläche herrschte eine Helligkeit von etwa 700 Lux. Die Wassertemperatur betrug zu Beginn des Versuchs $15^{\circ} \mathrm{C}$ und stieg im Laufe des Sommers auf ibber $18^{\circ} \mathrm{C}$ an (Abb. 2). Der Salzgehalt des benutzten Helgoländer Meerwassers betrug $35 \%$.

Das Becken wurde mit 180 Eiern versehen, welche am vierten Tag nach dem Ablaichen schlüpften. Unmittelbar nach dem Schlüpfen waren die Larven etwa 2,8 $\mathrm{mm}$ lang, während die Länge des Dottersacks $1 \mathrm{~mm}$ betrug. Die Larven trieben zunächst mit dem Dottersack nach oben passiv im Becken. Bereits am zweiten Tag nach dem Schlïpfen war der Dottersack weitgehend aufgezehrt, und erst jetzt waren die Augen voll pigmentiert. Die 4 bis $4,5 \mathrm{~mm}$ langen Larven hielten sich bevorzugt in Bodennähe auf; die meisten von ihnen begannen am dritten und vierten Tag mit der Nahrungsaufnahme. Als Nahrung wurden Nauplien des Salinenkrebses Artemia salina geboten. Es war auffällig, daß die Larven schon jetzt vorwiegend die in der Nähe des Bodens und an den Gefäßwänden sich aufhaltenden Nahrungsorganismen zu erbeuten suchten; hierbei wurde der Aufenthalt über hellem Untergrund eindeutig bevorzugt. Die Nahrungsdichte betrug in den ersten Tagen mindestens 20000 Nauplien pro Liter. Augenscheinlich ist dieses reichliche Nahrungsangebot wesentlich für den Aufzuchterfolg, da die Treffsicherheit der jungen Larven beim Beutefang zunächst nicht groß ist. Auch bei Coregonen sind zum Beispiel anfangs nur 3\% aller Beutefangreaktionen erfolgreich (Braum 1963). Die Unbeholfenheit der Artemia-Nauplien wirkte sich ebenfalls günstig auf den Aufzuchterfolg aus; denn es war zu beobachten, daß die Fischlarven, 
wenn sie auf die im Becken sich entwickelnden Harpacticiden gezielt Jagd machten, auf Grund der raschen Fluchtreaktionen dieser behenden Kleinkrebse nur selten zu einem Erfolg kamen. Die fast doppelt so großen Artemien wurden jedoch von ihnen ergriffen und aufgenommen. Nur bei Nahrungsmangel suchten die Larven auch die oberen Wasserschichten nach Futter ab. Die merkwürdig geringe Zahl von Seezungenlarven in den Planktonfängen war bereits älteren Autoren aufgefallen. (EHRENBAum 1905). Es erscheint sinnvoll, daß die sehr kompakten und von Anfang an stark pigmentierten Larven der Seezunge das Pelagial weitgehend meiden, wo sie gut sichtbar sind und für räuberisch lebende Arten eine leicht zu fangende Beute darstellen.

Am zehnten und elften Tage (etwa $6 \mathrm{~mm}$ Totallänge) wurden die Larven nahezu schlagartig rotbraun infolge des Auftretens zahlreicher braunroter, punktförmiger Chromatophoren in der Haut. Lediglich diejenigen, welche bis zu diesem Zeitpunkt noch keine Nahrung aufgenommen hatten, behielten die ursprüngliche, mehr grawe Larvenfärbung bei; sie starben $z$ wischen dem elften und zwölften Lebenstag zum größten Teil ab. Vom achtzehnten Tag an waren in zunehmendem Maße Larven (Totallänge 7 bis $7,5 \mathrm{~mm}$ ) $\mathrm{zu}$ beobachten, die sich, obwohl äußerlich noch völlig symmetrisch, in den Ruhepausen lateral auf den Boden legten; anscheinend wurde dabei keine Körperseite bevorzugt ${ }^{1}$. Zum Beuteerwerb, bei Fluchtreaktionen und ganz allgemein beim Schwimmen nahmen diese Larven jedoch stets die aufrechte Haltung eines normalen Fisches ein und suchten bei Nahrungsmangel auch gelegentlich höhere Wasserschichten auf. Schwierigkeiten, in normaler Schwimmlage die Balance zu halten, welche nach älteren Autoren eine Rolle in der Phylogenie der Plattfische gespielt haben sollen (Kyle 1923, Norman 1934), konnten nicht festgestellt werden. 22 Tage nach dem Schlüpfen - also erst mehrere Tage nach dem Einnehmen der Seitenlage - waren erste geringe Anzeichen der Augenwanderung festzustellen, die, einmal begonnen, sehr rasch fortschritt.

Am 25. Tag befand sich das blindseitige Auge bereits auf der dorsalen Kopfkante. Während dieses Auge sehr rasch und bei fast allen Individuen gleich schnell innerhalb von drei Tagen bis zum Scheitel wandert, verläuf die weitere Augenverlagerung bedeutend langsamer, und $z$ war bei den einzelnen Individuen mit unterschiedlicher Geschwindigkeit. Wenn das Auge die Kopfkante erreicht hatte, was bei einem Alter von 25 Tagen der Fall war, nahmen die Jungfische ihren ständigen Aufenthalt am Boden und behielten auch beim Fressen die Seitenlage bei; der Boden wurde jetzt auch während der Nahrungssuche im allgemeinen nicht mehr verlassen.

Im Alter von drei Wochen (Länge ungefähr $9 \mathrm{~mm}$ ) nahmen die Larven plötzlich eine schwarze Färbung an. Auf hellem Untergrund war auch bei starker Beleuchtung noch keinerlei farbliche Anpassung festzustellen. Bis zu diesem Stadium hatten sich die Larven gegenüber einer - beispielsweise durch Rühren erzeugten - Wasserströmung völlig passiv verhalten; sie krümmten sich allenfalls etwas ein, stellten die Schwimmbewegungen ein und ließen sich treiben. Auch nachdem das linke Auge den Scheitel erreicht hatte, ließen sie sich durch geringe Wasserströmungen ohne weiteres aufwirbeln.

Mit etwa dreißig Lebenstagen trat - bei einer Länge von ungefähr $15 \mathrm{~mm}$ - plötzlich eine Hellfärbung auf, welche an der Wurzel der unpaaren Flossen begann. Gleich-

1 Eine eingehende ethologische Untersuchung uber den vorliegenden Sachverhalt wurde von Hern cand. rer. nat. H. Rosentral durchgefultrt. 
zeitig mit dieser Umfärbung änderte sich das Verhalten gegenüber Wasserströmungen. Die Tiere reagierten nunmehr - ebenso wie die Adulten - mit Festklammern am Substrat. Zu diesem Zeitpunkt wurden in unserem Versuchsaquarium 152 Jungfische gezählt. Die Sterblichkeit bis zu diesem Entwicklungsstadium war mit knapp $20 \%$ recht niedrig und fast vollständig auf den elften bis dreizehnten Tag nach dem Schlüpfen beschränkt.

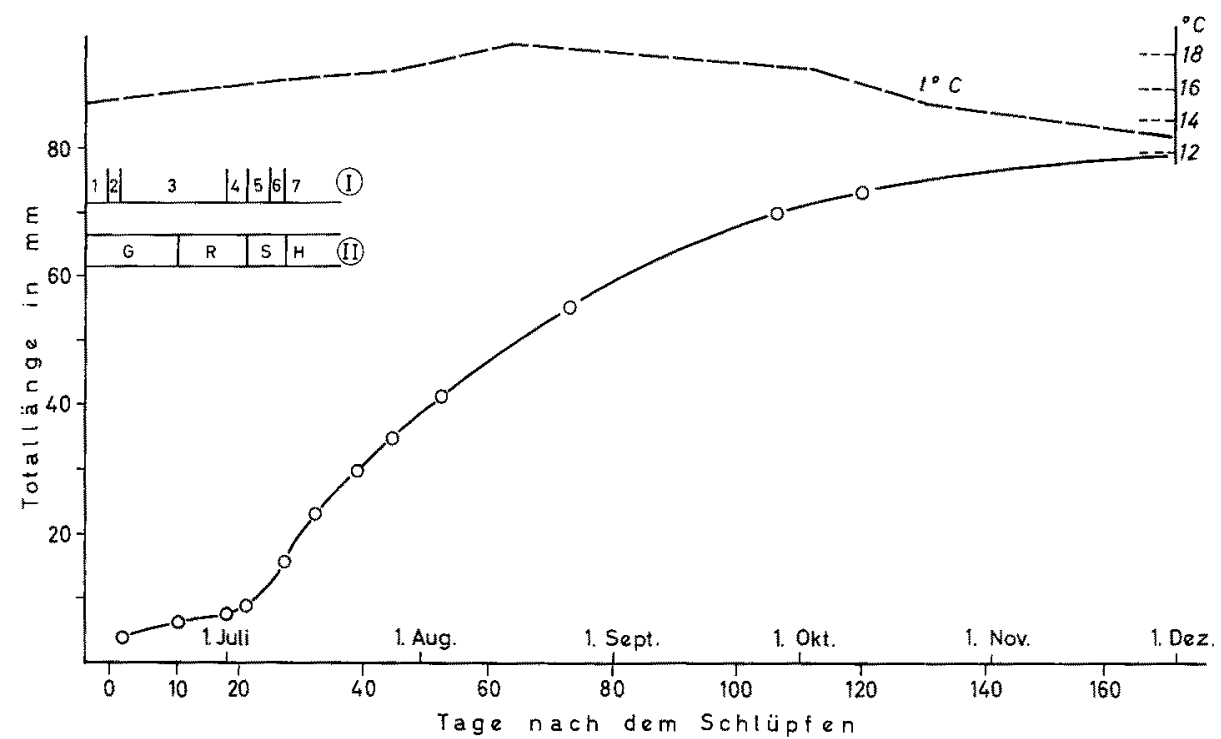

Abb. 2: Wachstumsverlauf von drei gut gefütterten Jungfischen (gemittelt). I: un terscheidbare "Entwicklungsstad ien", 1: Eistadium, 2: Dottersackstadium, 3: Beginn der Nahrungsaufnahme und Ablauf der üblichen Larvalentwidklung, 4: seitliches Liegen am Boden, 5: Beginn der Asymmetrie, 6: ständige Seitenlage, 7: Klammern am Substrat. II: We chs el der Pigmentierung während der Jugendentwicklung. G: graue Larvenfärbung, R: rotbraune Phase, S: schwärzliche Phase, H: "Aufhellung" und Fähigkeit zur farblichen Anpassung an das Substrat

$\mathrm{Da}$ es aus technischen Gründen nicht möglich war, alle Jungfische ausreichend zu ernähren, wurden dreißig Tage nach dem Schlüpfen drei Tiere in ein rundes, in gleicher Weise wie oben beschrieben, eingerichtetes Glas von $30 \mathrm{~cm}$ Durchmesser (gleiche Wassertemperatur) umgesetzt und kontinuierlich im Uberschuß mit Artemia salina-Larven versorgt. Diese drei Individuen wurden in der Folgezeit öfters gemessen; sie hatten nach sechs Monaten Längen von 85,79 beziehungsweise $73 \mathrm{~mm}$ erreicht (vgl. Abb. 2). Sechs Wochen nach dem Schlüpfen wurde ein zweites Mal eine Anzahl Seezungen aus dem Hauptbecken entnommen, in einen Kunststoffeimer aus Lupolen (am Boden etwa $20 \mathrm{~cm}$ Durchmesser) umgesetzt und ebenfalls reichlich ernährt; ihr Wachstum ist in Tabelle 1 wiedergegeben.

Vergleicht man die erreichten Längen mit den für das Wattenmeer angegebenen Daten (BückmanN 1934), so läßt sich feststellen, daß die Seezungen trotz der Kleinheit der Behälter bis zu diesem Zeitpunkt ebenso rasch gewachsen waren wie in ihrem natürlichen Habitat. Ein nennenswerter Einfluß der Behältergröße auf die Wachstumsintensität war nicht erkennbar. 
Tabelle 1

Längenzuwachs (Totallänge in $\mathrm{mm}$ ) von 5 Seezungen.

Wassertemperatur wie in Abbildung 2. M: Mittelwerte

\begin{tabular}{|cccc|}
\hline & 21. Juli & 27. September & 30. November \\
\hline & 20 & 70 & 82 \\
17 & 68 & 75 \\
17 & 66 & 75 \\
& 16,5 & 65 & 74 \\
& 16,5 & 63 & 70 \\
\hline$M$ & 17,4 & 66,4 & 75,2 \\
\hline
\end{tabular}

Das Wasser wurde im Hauptaquarium erstmalig nach sechs Wochen, später alle vier Wochen durch frisches ersetzt; in den anderen beiden Behältern erfolgte nur in zweimonatigen Abständen ein Wasserwechsel. Die im Hauptaquarium verbliebenen Fische wurden in ein- bis zweitägigen Abständen regelmäßig gefüttert. Das Nahrungsangebot war jedoch unzureichend, denn innerhalb einer Stunde waren alle Nahrungstiere aufgefressen. Diese Seezungen wuchsen nicht nur langsamer, sondern sie zeigten

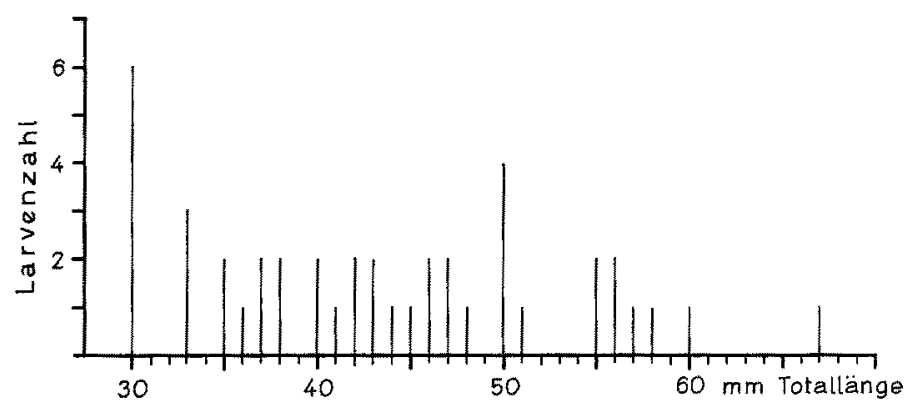

Abb. 3: Längenverteilung (Totallängen) der im "Hauptaquarium" verbliebenen unzureichend gef titterten Jungfische nach Ablauf von 6 Monaten

auch die von der künstlichen Aufzucht zahlreicher anderer Fische her bekannte Erscheinung des "Auseinanderwachsens". Die nach sechs Monaten erreichten Längen sind in Abbildung 3 wiedergegeben. Bei kontinuierlich im Überschuß angebotener Nahrung waren die Größendifferenzen erheblich geringer. In diesem Zusammenhang soll nicht unerwähnt bleiben, daß derartig langsam wachsende Jungfischbestände der Seezunge auch an natürlichen Standorten beobachtet wurden (APSTEIN 1904).

\section{ÜBER DEN EINFLUSS DER TEMPERATUR}

In Becken, die in gleicher Weise, wie oben beschrieben, eingerichtet und besetzt worden waren, aber täglichen Temperaturschwankungen zwischen $17^{\circ}$ und $20^{\circ} \mathrm{C}$ ausgesetzt waren, gelang es trotz reichlichen Nahrungsangebotes nicht, die Larven länger als 4 Tage am Leben zu erhalten. Die Sterblichkeit betrug unter diesen Bedingungen 
täglich etwa $30 \%$. Vermutlich wirkten die täglichen Schwankungen im Bereich dieser Temperaturen letal. Allerdings wuchsen Seezungen in Agypten bei Temperaturen von etwa $30^{\circ}$ heran (SAlaH EL-Din EL-Zarka 1963).

Beginnend mit dem 9. September wurde die Temperatur jeden zweiten Tag um $1^{\circ} \mathrm{C}$ herabgesetzt. Bei diesem allmählichen Absenken der Temperatur war keine augenfällige Schädigung der Jungfische zu beobachten. Noch bei $3^{\circ} \mathrm{C}$ wurden angebotene Salinenkrebse gierig gefressen. Erst bei $1^{\circ} \mathrm{C}$ wurde die Nahrungsaufnahme eingestellt. Ein zweitägiges Verbleiben bei $1^{0} \mathrm{C}$, dem ein plötzliches Ansteigen der Temperatur auf $16^{\circ} \mathrm{C}$ innerhalb von zehn Stunden folgte, wurde ohne Schädigung ertragen.

\section{ERBRÜTUNG UND AUFZUCHT DER FUTTERTIERE}

Die zunächst zur Fütterung verwendeten Nauplien von Artemia salina wurden bei $30{ }^{\circ} \mathrm{C}$ ebenfalls mit den beschriebenen Filterbecken aus Dauereiern erbrütet. Das Verfahren erleichterte vor allem die Gewinnung der ganz frisch geschlïpften Nauplien, die noch nicht positiv phototaktisch reagieren und sich zunächst am Boden aufhalten. Für die 9 bis $11 \mathrm{~mm}$ langen Jungfische, welche sich nicht mehr ins freie Wasser begaben, sondern nur in unmittelbarer Bodennähe Beute fingen, war die Versorgung mit diesem Naupliusstadium von entscheidender Wichtigkeit. Nach Erreichen einer Totallänge von $15 \mathrm{~mm}$ wurden die Seezungen zunächst mit etwa $4 \mathrm{~mm}$ Iangen Metanauplien, später mit adulten Artemia gefüttert, denn ihre rasch zunehmende Gefräßigkeit erschwerte eine Ernährung mit Nauplien erheblich. Enchyträen sowie feinzerteiltes Miesmuschelfleisch wurden nicht angenommen.

Zufällig stieß ich auf einen einzelligen grünen Flagellaten, Tetraselmis tetrabele West-BUTCHeR ${ }^{2}$, mit welchem es möglich war, Artemia salina vom Schlüpfen an innerhalb von zehn Tagen bis zum adulten Tier von etwa $15 \mathrm{~mm}$ Körperlänge heranzufüttern. Voraussetzungen für diese Anzucht sind Temperaturen von $25^{\circ}$ bis $30^{\circ} \mathrm{C}$ und intensive Beleuchtung von 20000 bis 30000 Lux. Die Algenkulturen waren nur dann zum Gedeihen zu bringen, wenn der Kulturlösung neben Natriumnitrat und Phosphat noch der Darminhalt von Seefischen beigemengt wurde. Da dessen Gewinnung mühsam ist, wurde nach einer anderen Substanz gleicher Wirkung gesucht. Es zeigte sich, daß außer Darminhalt von Seefischen auch "Heringslake" (die beim an Land erfolgenden Umpacken auf See eingesalzener Heringe anfallende Flüssigkeit) wirksam ist. Offensichtlich ist in diesen beiden heterogenen Substanzen ein Stoff enthalten, welcher für die Alge förderlich ist. Durch Kochen geht die Wirkung dieses unbekannten Stoffes nicht verloren. Mit der üblichen Erdabkochung, Muskulatur, Leber, Extrakten von beiden, roh oder gekocht, mit Hefe oder Hefeextrakt war keinerlei Wirkung zu erzielen.

Zur Massenaufzucht von Artemia salina wurde schließlich folgendes Verfahren entwickelt und für brauchbar befunden: unter freiem Himmel wurden vier Bretter von etwa $15 \mathrm{~cm}$ Breite rahmenartig aneinandergesetzt und der so gewonnene Kasten mit

2 Herrn Prof. Dr. H. A. von StosCH, Marburg, möchte ich herzlich danken für die Bestimmung des Flagellaten, welcher jetzt bei ihm in Reinkultur auf seine Lebensansprüche hin geprüft wird. 
Polyäthylenfolie ausgeschlagen. Es wurden zunächst Kästen von $1 \mathrm{~m}$ Breite und ungefähr 2,5 m Länge verwendet. (Die Tiefe entspricht der Breite der jeweils verwendeten Bretter.) Dem etwa $7 \mathrm{~cm}$ hoch eingefuillten Meerwasser wurde neben einem Kulturansatz des Flagellaten und den üblichen Nährsalzen noch etwa $2 \mathrm{ml}$ "Heringslake“ pro Liter zugesetzt. Wenn das Wasser sich dunkelgrïn färbte - im Hochsommer nach etwa zwei bis drei Tagen - wurden etwa 20000 Nauplien pro qm eingesetzt. Gegenüber Hineinregnen in die offenen Behälter und täglichen Temperaturschwankungen sind sowohl Artemia als auch Tetraselmis recht widerstandsfähig. Infolge des hohen Lichtund Wärmebedarfs konnte dieses Massenzuchtverfahren nur in der Zeit von Mai bis September erfolgreich betrieben werden.

\section{ZUSAMMENFASSUNG}

1. Eine Methode zur Brutaufzucht der Seezunge Solea solea (L.) wird beschrieben.

2. Sowohl unmittelbar in See gefangene als auch monatelang in Aquarien gehälterte Seezungen konnten zum Ablaichen gebracht werden. Ihre Larven wurden in kleinen Behältern mit Innenfiltern aufgezogen und mit Artemia-Nauplien ernährt. Die Sterblichkeit betrug bei $15^{\circ}$ bis $18^{\circ} \mathrm{C}$ ungefähr $20 \%$.

3. Die Larven sind gegenüber Temperaturschwankungen recht empfindlich. Vier Monate alte Jungfische nahmen jedoch bis zu Temperaturen von $3^{\circ} \mathrm{C}$ Nahrung auf und überlebten eine Abkühlung bis auf $1{ }^{\circ} \mathrm{C}$ ohne Schädigung.

4. Die Larven halten sich nach Aufzehrung des Dottersacks bevorzugt in Bodennähe auf. Außerlich noch völlig symmetrisch, nehmen sie zunächst die Seitenlage nur in den Ruhephasen ein, während beim Schwimmen und Beutefang die aufrechte Haltung beibehalten wird.

5. Im Verlauf der ersten fünf Wochen ändern die Larven mehrfach ihre Färbung. Anhand der jeweiligen Pigmentierung ist eine Unterscheidung der im Freien gefangenen Alters- und Entwicklungsstadien möglich. Mit Beginn der Hellfärbung im Alter von vier bis fünf Wochen setzt das von adulten Plattfischen her bekannte Anklammern am Substrat ein.

6. Die Wachstumsintensität in den verwendeten kleinen Behältern entsprach während der ersten sechs Monate den von BücKMaNN (1934) im Wattenmeer ermittelten Werten.

7. Ein Verfahren zur Massenkultur von Artemia salina mit dem als Futterorganismus besonders geeigneten Flagellaten Tetraselmis tetrabele wird mitgeteilt.

\section{ZITIERTE LITERATUR}

Apstern, C., 1904. Junge Butt (Schollen, Plewronectes platessa) in der Ostsee. Wiss. Meeresunters. N. F. Abt. Kiel 8, 1-25.

Braum, E., 1963. Die ersten Beutefanghandlungen junger Blaufelchen und Hechte. Z. Tierpsychol. 20, 257-266. 
Bücrmann, A., 1934. Untersuchungen über die Naturgeschichte der Seezunge, die Seezungenbevölkerung und den Seezungenfang in der Nordsee. Ber. dt. wiss. Komm. Meeresforsch. N. F. 7, 49-114.

Ehrenbaum, E., 1909. Eier und Larven yon Fischen. In: Nordisches Plankton. Hrsg. von K. Brandt \& C. Apstein. Zool. T. Lipsius \& Tischer, Kiel, Bd 1, 413 pp.

El-Zarka, Salar El-DIn, 1963. Acclimatization of Solea vulgaris (Linn.) in Lake Quarun, Egypt. J. Cons, perm. int. Explor. Mer 28, 126-136.

Fabre-Domergue, P. \& Bí́trix, E., 1905. Développement de la sole (Solea vulgaris). Vuibert \& Nony, Paris, 266 pp. (Trav, Lab. Zool. marit. Concarneau).

Fiǘchter, J., 1964. Eine besonders wirksame Aquarienfilterung und die Messung ihrer Leistung. Helgoländer wiss. Meeresunters. 11, 168-170.

KYLE, H. M., 1923. The asymmetry, metamorphosis and origin of flatfishes. Pbil. Trans, R. Soc, $211 \mathrm{~B}, 75-129$.

Norman, J. R., 1934. A systematic monograph of the flatfishes (Heterosomata). British Museum, London, Vol. 1, 459 pp. 